\title{
Simulation Analysis of Adsorption of Indoor Pollutants by Modified Activated Carbon
}

\author{
Sun Zhongxiang ${ }^{1}$ \\ ${ }^{1}$ Xi 'an University of Architecture and Technology, School of Building Services Science and Engineering, Xi 'an 710055, China
}

\begin{abstract}
As living standards greatly improves, people not only have higher demands for their own health, but also pay more attention to indoor air quality problems. Housing decoration will produce a large number of toxic and harmful pollutants, which will endanger the health of people. This paper mainly introduces the types and hazards of indoor pollutants and the purification methods of activated carbon. Taking the adsorption of formaldehyde as an example, the relationship between the improvement measures of activated carbon and the purification efficiency is studied through simulation calculation. The double-sided adsorption model was used as the carrier to study the influence of activated carbon modification on adsorption rate through theoretical analysis, which provided a reference for the improvement of activated carbon in actual production.
\end{abstract}

\section{Introduction}

Indoor decoration pollution refers to the pollution caused to the indoor environment during the decoration process. Indoor decoration pollutants include volatile organic compounds, radionuclides, strontium and other chemicals. Harmful gases that endanger human health are mixed into the indoor air, and people's respiratory and nervous systems are damaged. Usually these harmful substances come from paints, paints, plywood and insulation materials. Formaldehyde is commonly found in artificial boards of building materials. Formaldehyde emissions from urea-formaldehyde resin particleboard may last for months or even years [1], resulting in persistent pollution. An effective method is needed to remove formaldehyde. The influencing factors are analyzed by the model. At present, people mainly use natural plants and physical methods to remove indoor formaldehyde, and activated carbon adsorption as a kind of physical adsorption, almost no side effects, so the purification method in this paper chooses activated carbon adsorption. Activated carbon adsorption of formaldehyde is divided into static and dynamic adsorption. Hu Liuping [2] found that the efficiency of dynamic adsorption of formaldehyde can reach $90 \%$. When static adsorption occurs, the increase of airflow velocity is beneficial to adsorption. In actual life, due to various limitations such as temperature and wind speed, the adsorption capacity is insufficient, and the adsorption time is longer. However, the polarity of formaldehyde molecule is strong, and ordinary activated carbon has a weak adsorption capacity for formaldehyde [3], and its adsorption capacity can be improved by means of modification and the like. In this paper, the influence factors of adsorption process are analyzed based on the mass transfer coefficient of activated carbon. The influence degree of modified parameters of activated carbon is determined by analyzing the adsorption time model of formaldehyde, and a model reference is provided for the suitable modification range of activated carbon in actual production. The adsorption capacity of activated carbon can be improved by suitable modification measures with high economic efficiency.

\section{Sources and hazards of indoor pollutants}

Indoor air pollution can be divided into chemical pollution, physical pollution and biological pollution according to the types of pollutants. Among them, chemical pollution is mainly organic volatile compounds (VOCs), semiorganic volatiles (SVOCs), and harmful inorganic compounds. The pollution introduced during the renovation of the room is harmful to human health. Commonly used decoration materials are coatings, plywood, paint, plastics, insulation materials, etc., which will release pollutants such as formaldehyde, ammonia, benzene and so on. In this paper, formaldehyde was used as the main pollutant for removal analysis. Formaldehyde is mainly derived from various types of wood boards, coatings, etc. [1], the urea-formaldehyde resin is used as a binder in the production process, and the raw materials of the urine-aldehyde resin are formaldehyde and urea, and formaldehyde is slowly released during use. According to the GBT18883-2002 standard, the concentration of formaldehyde in residential buildings must be controlled below $0.08 \mathrm{mg} / \mathrm{m} 3$. The concentration of formaldehyde in the air exceeding $0.6 \mathrm{mg} / \mathrm{m}$ will irritate eyes and make the throat feel unsuitable for pain. Stay in the air containing $10 \mathrm{ppm}$ of formaldehyde for a few minutes, and the eyes will shed tears. Because formaldehyde binds to proteins, high concentrations of 
formaldehyde can cause severe respiratory irritation, edema and headaches [4].

At present, indoor formaldehyde removal methods mainly include plant purification and activated carbon adsorption. Wei Haifeng et al. [5] showed that when 10 $\mathrm{mL}$ of $40 \%$ formaldehyde solution in the culture dish was also used, the effect of the first two days of clivia was not significant under closed environmental conditions. When the eighth day was reached, the effect gradually stabilized and the removal rate was obtained as high as $77 \%$. The removal effect of activated carbon is better, and the removal efficiency can reach $82.6 \%$ in seven days.

\section{Activated carbon adsorption technology and modification}

Plant adsorption efficiency is low and cannot be used as the main purification method. Physical adsorption is a simple and feasible method without any side effects and by-products. Activated carbon adsorption is the most widely used method. Activated carbon is a porous substance made of carbon-containing materials through high-temperature carbonization and activation. It has rich pore structure, large specific surface area, strong adsorption capacity and great adsorption capacity [6]. The preparation of activated carbon is relatively easy. It can be made from almost all carbon-containing substances such as coal, wood, fruit shell and so on. Different types of activated carbon can be used in different situations. Activated carbon adsorption is mainly used to treat common organic compounds, including benzene, formaldehyde and other gases.

Liu Chao et al. [7] showed that there are three ways to modify activated carbon: oxidation modification, reduction modification and loading metal modification. Oxidative modification means that the activated carbon indicates that more oxygen-containing functional groups are produced, the acid strength of the activated carbon is increased, and more polar compounds are adsorbed [8]. The reduction modification produces more surface oxygen-containing basic functional groups and hydroxyl functional groups, and increases the surface non-polarity to adsorb more non-polar compounds. The modification of supported metal can change the performance of activated carbon from physical adsorption to chemical adsorption, which improves the adsorption capacity.

\section{Simulation calculation}

In the modeling and analysis of activated carbon adsorption, it is necessary to consider the installation position of activated carbon. In a cubic room, point adsorption or surface adsorption can be set, however, point adsorption is difficult to calculate. In this paper, surface adsorption method is used for simulation analysis, single-sided adsorption. The time required is longer, so after analyzing the single-sided adsorption, a doublesided adsorption model is established, which can meet the adsorption requirements in a suitable adsorption time. We simplify the complex room shape into a cube to simulate the adsorption process of activated carbon in a square room, regardless of the position of the partition wall in the middle. The programming calculation software used in the experiment is Matlab 2018.

The simplified conditions of the model are as follows:

1. We set the size of the bedroom in the home to $5 \mathrm{~m} * 5 \mathrm{~m} * 2.5 \mathrm{~m}$

2. The initial formaldehyde concentration in the environment is high, and the window is ventilated to reduce the concentration to $1 \mathrm{mg} / \mathrm{m} 3$. China National Standard "Sanitary Standards for Formaldehyde in the Air of the Room" stipulates that the maximum allowable concentration of formaldehyde in the living room air is $0.08 \mathrm{mg} / \mathrm{m} 3$, and the adsorption target is to reduce the ambient formaldehyde concentration to $0.08 \mathrm{mg} / \mathrm{m} 3$.

3 . The relative density of formaldehyde gas is 1.067 . So it will lead to a sedimentation in the room. In this problem, the deposition of gas molecules is not considered for the time being. It is considered that formaldehyde in air is uniformly distributed under initial conditions.

4. The air temperature is $25{ }^{\circ} \mathrm{C}$, ignoring the adsorption thermal effect, maintaining atmospheric pressure and constant humidity.

5. We use an excess of uniformly dense activated carbon.

For the above environment setting, the following model is used to simulate the adsorption process in the room. The calculation process refers to the analysis process of the non-steady-state heat conduction part in the book [9].

1. Evenly distribute activated carbon on one side (floor)

We assumed that the adsorption capacity of activated carbon is very powerful, the concentration of formaldehyde on the surface is 0 , and the indoor environment is simplified to a semi-infinite plate adsorption model that ignores the surface resistance, and the formaldehyde concentration at the ceiling is reduced to the standard below to achieve the adsorption purpose. The space right-angle coordinate system is established with the center of the floor, and the y-axis direction is the center of the floor pointing to the center of the ceiling. From the symmetry, only the mass transfer on the y-axis will be considered.

The mass transfer differential equation for this process is:

$$
\frac{\partial \mathrm{c}}{\partial \mathrm{t}}=\mathrm{D}_{\mathrm{AB}} \frac{\partial^{2} \mathrm{c}}{\partial \mathrm{y}^{2}}
$$

$D_{A B}$ is the diffusion coefficient of formaldehyde in air. Initial condition:

$\mathrm{t}=0, \mathrm{y} \gg 0, \mathrm{c}=\mathrm{c}_{0}=1 \mathrm{mg} / \mathrm{m}^{3}$

Boundary conditions:

$$
\begin{aligned}
& \text { (1) } t>0, y=0, c=c_{w}=0 \\
& \text { (2) } t=t_{f}, y=2.5, c=c_{1}=0.08 \mathrm{mg} / \mathrm{m}^{3}
\end{aligned}
$$

$\mathrm{t}_{\mathrm{f}}$ The time required for the contaminant to fall from $\mathrm{c} 0$ to $\mathrm{c} 1$.

Let variable $\mathrm{N}$ be:

$$
\mathrm{N}=\frac{\mathrm{y}}{2 \sqrt{\mathrm{D}_{\mathrm{AB}} \mathrm{t}}}
$$

The original differential equation is:

$$
\frac{\partial^{2} \mathrm{c}}{\partial \mathrm{N}^{2}}+2 \mathrm{~N} \frac{\partial \mathrm{c}}{\partial \mathrm{N}}=0
$$

The initial condition becomes: 
$\begin{aligned} \text { When } \mathrm{t}=0, \mathrm{y} \gg & 0(\text { a Limited value }) \text {, i. e. } \mathrm{n} \rightarrow \infty, \mathrm{c} \\ & =\mathrm{c}_{0}=1 \mathrm{mg} / \mathrm{m}^{3}\end{aligned}$

The boundary condition becomes:

(1) $t>0, y=0$, that is, when $N=0, c=c_{w}=0$

(2) $\mathrm{t}=\mathrm{t}_{\mathrm{f}}, \mathrm{y} \gg 0$, i.e. $\mathrm{n}=\frac{\mathrm{y}}{2 \sqrt{\mathrm{D}_{\mathrm{AB}} \mathrm{t}_{\mathrm{f}}}}, \quad \mathrm{c}=\mathrm{c}_{1}=$ $0.08 \mathrm{mg} / \mathrm{m}^{3}$

Integrate the above differential equation to:

$$
\frac{\mathrm{dc}}{\mathrm{dN}}=\mathrm{C}_{1} \mathrm{e}^{-\mathrm{N}^{2}}
$$

Integration after substituting boundary conditions and initial conditions:

$$
\rho=\frac{2}{\sqrt{\pi}} \int_{0}^{\mathrm{N}} \mathrm{e}^{-\mathrm{N}^{2}} \mathrm{dN}=\operatorname{erf}(\mathrm{N})
$$

Substituting boundary conditions (2), i.e.

$$
\mathrm{c}=\operatorname{erf}(\mathrm{N})=0.08
$$

The error function calculated by Matlab is as follows:

$$
\mathrm{N}=0.071
$$

The diffusion coefficient of formaldehyde in air is calculated by the semi-empirical formula of Gilliland.

$$
\mathrm{D}_{\mathrm{AB}}=1.393 \times 10^{-5} \mathrm{~m}^{2} / \mathrm{s}
$$

byN $=\frac{\mathrm{y}}{2 \sqrt{\mathrm{D}_{\mathrm{AB}} \mathrm{t}_{\mathrm{f}}}}=0.071$ When $\mathrm{y}=2.5$, the concentration of formaldehyde in the room is less than $0.08 \mathrm{mg} / \mathrm{m}^{3}$, on behalf of the time:

$$
\mathrm{t}_{\mathrm{f}}=257.5 \text { day }
$$

From the calculation results, the time that indoor formaldehyde concentration drops below standard value is too long In order to investigate the rationality of the model, the model Fourier number is calculated:

$$
\mathrm{F}_{\mathrm{O}}=\frac{\mathrm{D}_{\mathrm{AB}} \mathrm{t}_{\mathrm{f}}}{\mathrm{L}^{2}}=49.6 \gg 0.1
$$

After calculation, it is found that the Fourier number of the model is much larger than 0.1 , indicating that the mass transfer process is simplified to the mass transfer of the non-resistance semi-infinite medium with great error, which will not be considered in the following discussion.

2. Evenly distribute activated carbon on two opposite sides (for example, floor and ceiling)

We assumed that the adsorption capacity of activated carbon is very powerful, the concentration of formaldehyde on the surface of the activated carbon is 0 , which simplifies the indoor environment to a semi-infinite plate adsorption model that ignores surface resistance. The highest formaldehyde concentration in the room is less than the standard below the task to achieve the purpose of adsorption.

The space right-angle coordinate system is established in the center of the room. The positive half-axis direction of the $y$-axis is the center of the floor pointing to the center of the ceiling. From the symmetry, only the mass transfer on the y-axis can be considered.

The mass transfer differential equation for this process is:

$$
\frac{\partial \mathrm{c}}{\partial \mathrm{t}}=\mathrm{D}_{\mathrm{AB}} \frac{\partial^{2} \mathrm{c}}{\partial \mathrm{y}^{2}}
$$

Among them $\mathrm{D}_{\mathrm{AB}}$ is the diffusion coefficient of formaldehyde in the air.

The initial conditions are:

$$
\mathrm{t}=0, \mathrm{y} \gg 0, \mathrm{c}=\mathrm{c}_{0}=1 \mathrm{mg} / \mathrm{m}^{3}
$$

The boundary conditions are:

$$
\begin{aligned}
& t>0, y=0, \frac{\partial c}{\partial y}=0 \\
& t=t_{f}, y=1.25, c=c_{1}=0 \mathrm{mg} / \mathrm{m}^{3}
\end{aligned}
$$

The solution is obtained at a given $\mathrm{y}$ value with the following concentration distribution:

$$
\frac{\mathrm{c}(\mathrm{y})-\mathrm{c}_{1}}{\mathrm{c}_{0}-\mathrm{c}_{1}}=\sum_{\mathrm{n}=1}^{\infty} \frac{4(-1)^{\mathrm{n}+1}}{(2 \mathrm{n}-1) \pi} \cos \left[\left(\frac{2 \mathrm{n}-1}{2}\right) \pi \frac{\mathrm{y}}{\mathrm{L}}\right] \mathrm{e}^{-\left(\frac{2 \mathrm{n}-1}{2} \pi\right)^{2} \frac{\mathrm{D}_{\mathrm{AB}} \mathrm{t}}{\mathrm{L}^{2}}}
$$

In the above formula, $\mathrm{L}$, half of the room height, is $1.25 \mathrm{~m}$. The solution is that when $\mathrm{t}=1.46$ days, the concentration of the center of the $\operatorname{room}(\mathrm{y}=0)$ is 0.08 $\mathrm{mg} / \mathrm{m}^{3}$, which achieves the expected adsorption effect. In order to verify the accuracy of the model, the image of formaldehyde concentration values under different time and height conditions is plotted as shown in Fig. 2. When $\mathrm{t}=0$ in the image, the concentration value is a straight line, which meets the initial setting conditions. With the increase of adsorption time, the concentration decreases gradually in all altitudes. The closer the adsorbent is, the faster the concentration of adsorbent decreases, which meets the hypothetical conditions.

At a certain height, the concentration of formaldehyde decreases with time. Moreover, the concentration of formaldehyde at the height of the center of the room is always the highest at a certain time. After 1.46 days of adsorption, the adsorption requirements were met.

Through the above model analysis, considering that the adsorption saturation time of commonly used activated carbon is 8-15 days, the purpose of adsorption can be achieved within an acceptable data, 1.46 days. The double-sided adsorption phase is greatly improved compared to the single-sided adsorption efficiency, and can be applied to practice. At the same time, mass transfer resistance exists in the actual adsorption process. In the subsequent analysis, it is necessary to consider the influence of surface mass transfer resistance on the adsorption time to describe the real adsorption process more accurately.

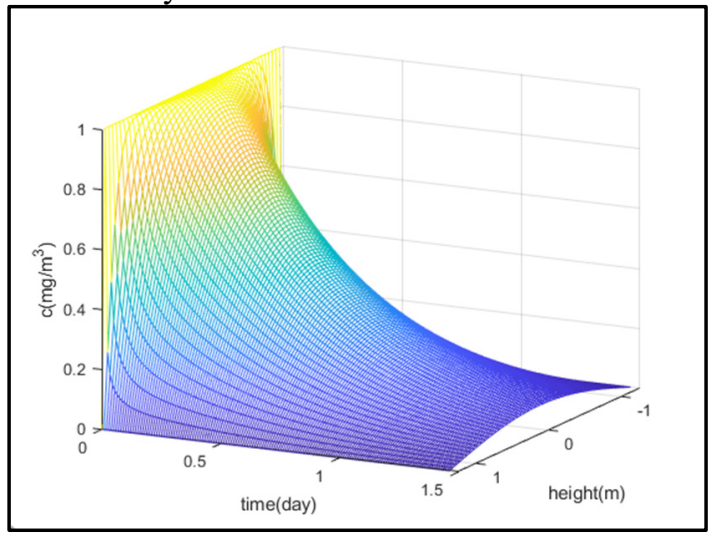

Fig.1. Formaldehyde concentration under different height and time conditions without mass transfer resistance

3. On the basis of model 2, we then consider the effect of surface mass transfer resistance on adsorption time. Under this condition, the boundary conditions of the mass transfer model change, which means that the formaldehyde molecules cannot be adsorbed by the activated carbon instantaneously, and the concentration value at the boundary changes with time. Considering the influence of the resistance, the real process can be described more accurately, and the influence of the resistance on the adsorption rate is analyzed, which also has a certain guiding effect on the application of activated carbon in the real process. 
In the setting conditions of Model 2, considering the mass transfer resistance, the mass transfer differential equation of this process is:

$$
\frac{\partial \mathrm{c}}{\partial \mathrm{t}}=\mathrm{D}_{\mathrm{AB}} \frac{\partial^{2} \mathrm{c}}{\partial \mathrm{y}^{2}}
$$

among them $\mathrm{D}_{\mathrm{AB}}$ It is the diffusion coefficient of formaldehyde in the air.

The initial conditions are:

$$
\mathrm{t}=0, \mathrm{y} \gg 0, \mathrm{c}=\mathrm{c}_{0}=1 \mathrm{mg} / \mathrm{m}^{3}
$$

The boundary conditions are:

$$
\begin{aligned}
& t>0, y=0, \frac{\partial c}{\partial y}=0 \\
& t=t_{f}, y=1.25, K k\left(c-c_{\infty}\right)=-D_{A B} \frac{\partial c}{\partial y}
\end{aligned}
$$

The solution is obtained at a given $y$ value with the following concentration distribution:

$$
\frac{c(y)-c_{\infty}}{c_{0}-c_{\infty}}=2 \sum_{n=1}^{\infty} \frac{\sin \mu_{n} \cos \mu_{n}\left(\frac{y}{L}\right)}{\mu_{n}+\sin \mu_{n} \cos \mu_{n}} e^{-\left(\mu_{n}\right)^{2} \frac{D_{A B} t}{L^{2}}}
$$

In the above formula, $c_{\infty}$ Under the equilibrium adsorption model of activated carbon, the value was still determined to be zero.Since the intermediate calculation process is more complicated, the intermediate value is given here. $\mu_{\mathrm{n}}$ Expression:

$$
\begin{aligned}
& \cot \left(a_{n} L\right)=\frac{D_{A B} a_{n}}{K k} \\
& \mu_{n}=a_{n} L
\end{aligned}
$$

In the above formula, $\mathrm{k}$ is the convective mass transfer coefficient of the surface of the activated carbon, and $\mathrm{K}$ is the equilibrium constant. The physical meaning is the ratio of the formaldehyde concentration on the surface of the activated carbon to the concentration of formaldehyde in the surface layer of the activated carbon. The factors affecting the convective mass transfer coefficient are numerous and complex, depending on the physical properties of the fluid, the shape arrangement of the mass transfer surface, etc. The factors that are most important in determining the various parameter values are solved by experiments. In this paper, the above two parameters are analyzed by empirical values. In general, the convective mass transfer coefficient $\mathrm{k}$ and the diffusion coefficient $\mathrm{D}$ satisfy the following relationship:

$$
\mathrm{k}=\mathrm{D}^{0.5 \sim 1.0}
$$

In this paper, we use Matlab to solve one of the special cases of adsorption problem. When $\mathrm{K}=2$ and $\mathrm{K}=\mathrm{D}^{0.8}$, the time to reach the adsorption goal is 1.58 days, and the central concentration is $0.08 \mathrm{mg} / \mathrm{m}^{3}$. Fig. 2 is a graph showing the concentration of formaldehyde at different heights at different times after the code is added:

Considering the actual mass transfer resistance, compared with Fig. 1, Fig.2 takes longer to achieve the adsorption purpose. In the case of $y= \pm L$, In Fig.2, the boundary concentration decreases gradually from the initial condition. In Model 2, the boundary concentration is always 0 , which also shows that Model 3 is more in line with the actual conditions.

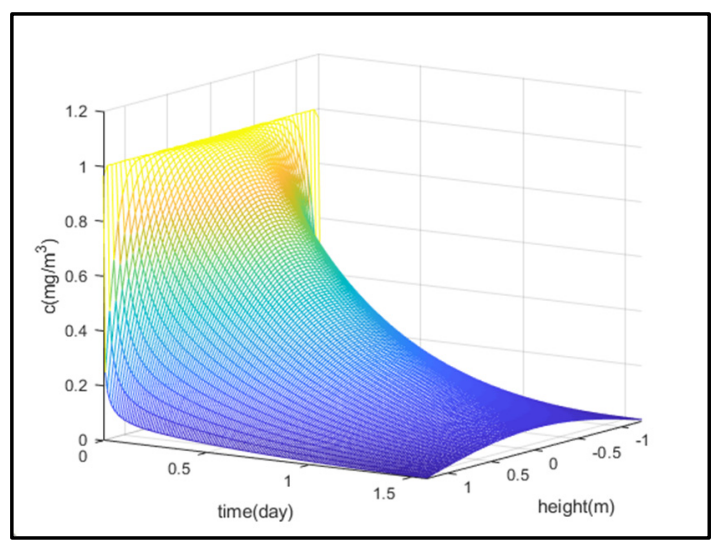

Fig.2. Formaldehyde concentration under different heights and time conditions with mass transfer resistance

Considering the degree of simplification of the model and the mass transfer resistance in practical application, a more in-depth parameter analysis is performed for the double-sided adsorption model. In fact, the parameters that directly affect the adsorption time include mass transfer rate, initial concentration, room height, etc. In a certain system, changing the parameters of activated carbon affects $\mathrm{k}$ and $\mathrm{K}$, and has a direct impact on the adsorption time. In order to analyze the influence of the above two parameter analysis on the adsorption time, analyze the parameters in the range of $\mathrm{K} \sim[0.5,2]$ and $\mathrm{k} \sim\left[\mathrm{D}^{0.9}, \mathrm{D}^{0.7}\right]$. The data of the time taken for the adsorption when the parameter is changed is obtained by calculation. The following images can be obtained by plotting the time between $\mathrm{k}$ and $\mathrm{K}$ :

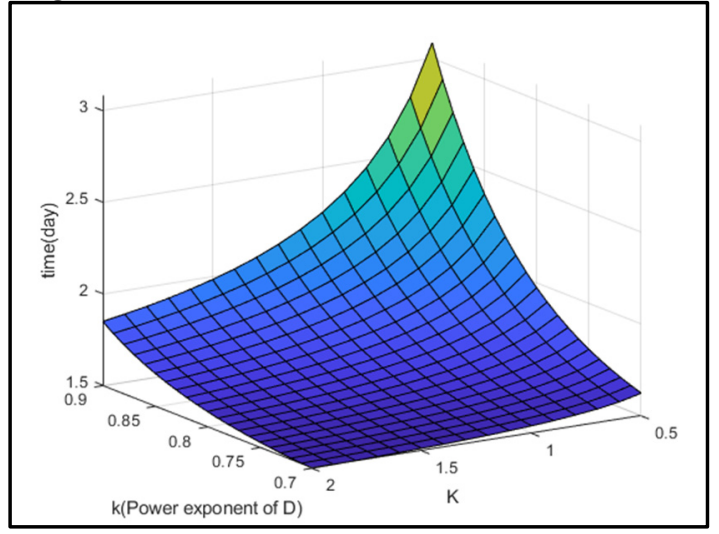

Fig.3 Adsorption time under different $\mathrm{K}$ and $\mathrm{k}$ parameters

From the Fig.3 analysis, if the mass transfer coefficient $\mathrm{k}$ remains unchanged, the adsorption time increases with the decrease of equilibrium constant $\mathrm{K}$; under the condition that the $\mathrm{K}$ is constant, the adsorption time increases with the decrease of $\mathrm{k}$. The conclusion is drawn that the larger the equilibrium constant $\mathrm{K}$ and the larger the mass transfer coefficient $\mathrm{k}$, the more favorable the adsorption of formaldehyde gas and the shorter the adsorption time.

In order to analyze the influence of the above two parameters on the adsorption time, the parameter gradient analysis of Fig. 4 is carried out to obtain the change rate of adsorption time under different parameters to find suitable and effective parameter pairs in specific applications. The data image of the gradient analysis is 
shown in Fig. 4:

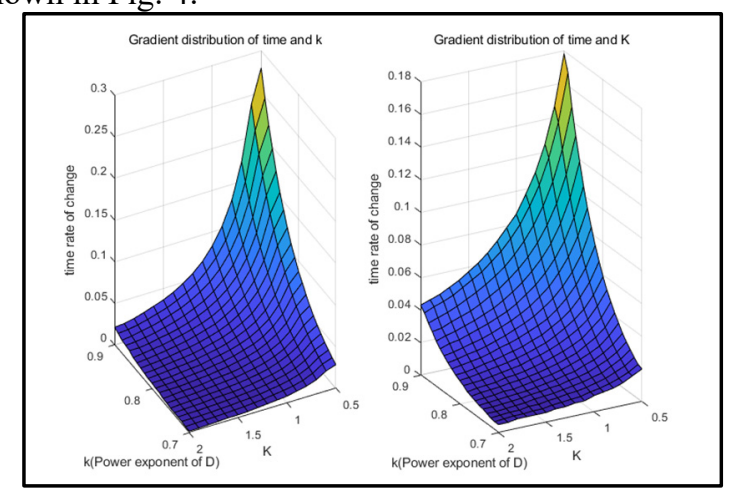

Fig.4 Gradient analysis of influencing factors of adsorption time

Under the condition of constant mass transfer coefficient $\mathrm{k}$, with the decrease of equilibrium constant $\mathrm{K}$, the change rate of adsorption time increases. It is concluded that when the equilibrium constant $\mathrm{K}$ and mass transfer coefficient $\mathrm{k}$ are large, their changes have little effect on the growth rate of adsorption time. On the contrary, the influence is greater when they are smaller.

The main influencing factors of $\mathrm{k}$ are the pore size, wind speed, temperature, humidity and modification of activated carbon. Since the indoor temperature, humidity and wind speed depend on the local climate, the effect of modification on mass transfer coefficient $\mathrm{k}$ is mainly discussed here. Liu et al. [10] found that the improvement of adsorption performance of acid modification is stronger than that of alkaline modification, so we focus on the analysis of acid(oxidation) modification. After $\mathrm{HNO}_{3}$ oxidation modification of activated carbon, Liu Wenhong et al. [11] found that the oxygen-containing groups on the surface of activated carbon increased, and the adsorption active sites increased, which improved the adsorption capacity of carbon atoms in formaldehyde molecules and the mass transfer coefficient. Hu et al. [3] Hu used $\mathrm{KMnO}_{4}$ to oxidize the activated carbon from fruit shell. It was found that the adsorption capacity was 10 times higher than that of the original activated carbon. However, when Fan et al. [12] modified activated carbon with $\mathrm{HNO}_{3}$, it was found that the structure of activated carbon was destroyed, the specific surface area was reduced and adverse effects would be produced while the adsorption capacity was increased.

From the simulation results and literature research, oxidation modification can effectively improve the mass transfer coefficient and enhance the adsorption performance. In practical application, the size of the two parameters should be appropriately increased in consideration of the influence of the gradient, that is, the modified activated carbon with more economical parameter range should be prepared under the condition of satisfying the purpose of adsorption.

\section{Conclusions}

In summary, we have established a double-sided adsorption model with surface resistance. It is found that when the mass transfer coefficient and equilibrium constant are in a lower range, the amount of change has a greater influence on the change rate of adsorption time and when they are larger, the amount of change has little effect on the change rate of adsorption time. Therefore, for the modification of activated carbon, the determination of the above two parameters in a suitable range can ensure the adsorption capacity and determine the appropriate degree of modification, which is a more economical method for the production of modified activated carbon. For residents, in the process of air purification after indoor decoration, priority should be given to using natural ventilation to dilute pollutants and use more environmental-friendly decoration materials. Meanwhile, we use qualified activated carbon indoors, so that human health can be better guaranteed.

\section{References}

1. Salthammer T, Mentese S, Marutzky R. 2010. Formaldehyde in the Indoor Environment. Chemical Reviews 110: 2536-72

2. Hu Liuping, Mo Kailin, Yang Ling, Zhang Bi. Research on Activated Carbon to Adsorption of formaldehyde [j]. Sichuan Forestry Science and Technology, 2007 (04): 52-54+15.

3. Hu S, Chen Y, Lin X, Shiue A, Huang P, et al. 2018. Characterization and adsorption capacity of potassium permanganate used to modify activated carbon filter media for indoor formaldehyde removal. Environmental Science and Pollution Research 25: 28525-45

4. Tanada S, Kawasaki N, Nakamura T, Araki M, Isomura M. 1999. Removal of Formaldehyde by Activated Carbons Containing Amino Groups. $J$ Colloid Interface Sci 214: 106-8

5. Wei Haifeng, Zhao Xiaoyi, Yu Haiping, Cao Yang, Li Xiangyang. Study of the Effects of Several Methods on Formaldehyde $\mathrm{R}$ emoval [j]. Environmental Protection Science, 2018, 44 (05): 73-76.

6. Lee KJ, Miyawaki J, Shiratori N, Yoon S, Jang J. 2013. Toward an effective adsorbent for polar pollutants: Formaldehyde adsorption by activated carbon. Journal of Hazardous Materials 260: 82-8

7. Liu Chao, Yu Hui, Yu Qingyue. Research progress in activated carbon modification methods [j]. Chemical Engineering and Equipment, 2018 (02): 253-254.

8. Houshmand AH, Daud WMAW. 2010. Study of Changes in a palm-shell-based Activated Carbon Characteristics by Nitric Acid. Journal of Applied Sciences 10: 1116-21

9. Geankoplis, Christie J. Transport processes and separation process principles:(includes unit operations). Prentice Hall Professional Technical Reference, 2003.

10. Liu Xiuyu, Zhang Hao. Adsorption Performance of Formaldehyde Gas onto Modified Biomass Activated Carbon [j]. Non-metallic minerals, 2019, 42 (04): 8285. 
11. Liu Wenhong, Yuan Huaibo, Lu Jianping. Effect of modification of activated carbon with $\mathrm{HNO} 3$ at different temperatures on silver adsorption [j]. Chinese Journal of Nonferrous Metals, 2007(04): 663-667.
12. Fan Yanbiao, Wang Baozhen, Wang Lin, Yu Min. Surface Characteristics of Modified Activated Carbon and Its Adsorption Properties for Metal Ions[J]. Environmental Chemistry, 2001(05): 437443. 